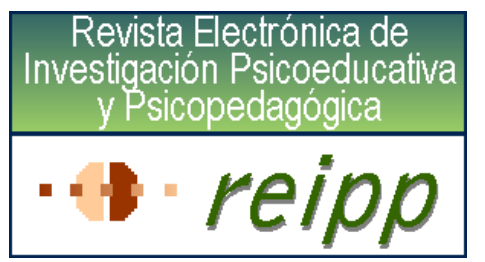

REVISTA ELECTRÓNICA DE

INVESTIGACIÓN PSICOEDUCATIVA Y

PSICOPEDAGÓGICA

No 1 (2) 2003. ISSN: 1696-2095

\title{
Aproximación a una formación académica de calidad: el punto de vista de los estudiantes universitarios
}

\author{
Ignacio González López
}

Departamento de Ciencias de la Educación. Área MIDE.

Universidad de Córdoba

España

ed1goloi@uco.es 


\section{RESUMEN}

Introducción. El objetivo prioritario del trabajo que aquí se propone se centra en definir una universidad de calidad desde la perspectiva del usuario, a partir de las manifestaciones planteadas por los alumnos en diferentes aspectos institucionales, comprobando la coherencia de sus opiniones bajo dos formatos metodológicamente diferentes de recogida de información y contrastando los resultados obtenidos desde un planteamiento tanto cualitativo como cuantitativo en su respuesta.

Método. Aplicada una encuesta de opinión a una muestra de 807 estudiantes universitarios y sobre un modelo de relación de variables entrada-proceso-producto, procedimos a llevar a cabo un estudio de las respuestas aportadas por medio de técnicas de análisis cuantitativo (estudios descriptivo, inferencial, correlacional y multivariante) y de análisis cualitativo (análisis de contenido).

Resultados. Tras la aplicación de la técnica del análisis de contenido, hemos podido advertir cuál ha sido la tendencia en la respuesta de los estudiantes encuestados, advirtiendo su compromiso con el instrumento de recogida de información, así como los rasgos que, desde su perspectiva, conforman una buena formación. Se han hecho patentes los males que la afectan y se han propuesto alternativas para su mejora. Al final, hemos obtenido datos para describir los elementos que conforma una Universidad de calidad. Estas manifestaciones se han visto validadas tras un estudio de regresión múltiple donde, el aspecto que mejor representa la calidad de una Universidad es la satisfacción del alumnado, un indicador de producto, manifestación de la política educativa actual en materia de evaluación institucional.

Discusión. Con este trabajo hemos podido constatar la correcta compatibilidad de técnicas de análisis cualitativo y cuantitativo de datos en la realización de estudios como el que aquí se ha presentado. Al mismo tiempo, las cuestiones aquí planteadas han venido a ser un referente donde los estudiantes han podido satisfacer la necesidad de dar una opinión crítica sobre el sistema universitario del que forma parte.

Palabras-clave: análisis cualitativo de datos, análisis cuantitativo de datos, evaluación educativa, universidad de calidad, satisfacción del alumnado 


\section{Introducción}

El tema de la calidad no es una novedad, sino que es recurrente en la investigación educativa en un contexto internacional (De la Orden, 1988; De Miguel y Rodríguez Espinar, 1991; Doherty, 1994; Quintanilla, 1998; López Mojarro, 1999; Pérez Juste et al., 2000; Cantón Mayo, 2001; MECD, 2001; EFQM, 2002). Sin embargo, ha adquirido una importancia relevante dentro de la legislación educativa actual, particularmente en el ámbito universitario. En este nivel, la promoción de la calidad asociada a todos los elementos constitutivos de una institución, como exigencia social, es el principal objetivo de la Ley Orgánica de Universidades (2001).

Pero su interpretación es muy diferente dependiendo de los agentes que la formulen y de la metodología empleada, no sólo para alcanzarla, sino producirla. Por esta razón, la Unión Europea ha adoptado un modelo de evaluación (EFQM) que integra la lógica de los modelos existentes en Japón (Deming) y Estados Unidos (Baldrige). Se trata de un modelo integrado plenamente en las instituciones educativas españolas que incorpora la lógica del análisis estadístico al control de la calidad del modelo japonés y asume el objetivo de satisfacer a los usuarios, óptica del modelo americano.

Sin embargo, según afirma Tejedor (2003), la preocupación hoy ya no es cuántos alumnos reciben educación y en qué proporción, sino quienes aprenden, qué aprenden y en qué condiciones aprenden. En definitiva, el gran reto de la educación para este nuevo siglo es la búsqueda de la calidad (OCDE, 1991; Marchesi y Martín, 1998 y Gazïel et al., 2000), relacionada de forma directa con la satisfacción del alumnado, al que se ha de proporcionar una educación completa a través de metodologías didácticas innovadoras, por medio del empleo eficaz de los recursos y transmitiendo contenidos conceptuales, procedimentales y actitudinales, que le permitan desarrollarse como ser social, tanto a escala profesional como personal.

La búsqueda y promoción de la calidad en educación ha de constituirse como un proceso reflexionado, sistematizado y consensuado por parte de todos los miembros de la comunidad educativa, con el objetivo de llevar a cabo la evaluación de la situación en la que tienen lugar los diferentes fenómenos educativos. Esto dará lugar al descubrimiento de las potencialidades de la institución y de los elementos que la constituyen, ayudará a identificar las debili- 
dades y potenciará el desarrollo de propuestas innovadoras que ejecuten el cambio deseado y consigan un desarrollo continuo y constante, tanto de la organización como de sus miembros.

\section{Objetivos del estudio}

El objetivo prioritario del trabajo que aquí se propone se centra en definir una Universidad de calidad desde la perspectiva del usuario, a partir de las manifestaciones planteadas por los alumnos en diferentes aspectos institucionales, comprobando la coherencia de sus opiniones bajo dos formatos metodológicamente diferentes de recogida de información y contrastando los resultados obtenidos desde un planteamiento tanto cualitativo como cuantitativo en su respuesta.

\section{Método}

\section{Muestra}

Para realizar este estudio seleccionamos una muestra de 807 estudiantes aplicando un criterio de proporcionalidad, en base a la rama de especialización a la que pertenecen los estudios en que se encuentran matriculados: Ciencias de la Salud, Humanidades, Experimentales, Jurídico-Sociales y Técnicas.

\section{Procedimiento}

Una vez se formularon los objetivos, procedimos a especificar las variables que daban cuenta de los fenómenos a estudiar. Para su selección hemos considerado aquellos factores que condicionan la calidad de una institución universitaria. Basándonos en una estructura relacional que clasifica las variables en entrada, proceso y producto, trabajamos con un total de cincuenta y cinco, reflejadas en la figura 1.

Observamos que las variables de entrada recogen los aspectos básicos que describen al alumnado en cuanto a su identificación personal y académica, así como otros factores de clasificación y condicionantes de la realización de estudios universitarios. Al mismo tiempo, han sido tratados todos aquellos aspectos relacionados con las actitudes del alumnado de nuevo ingreso hacia la institución en la que cursan sus estudios. 
Figura 1: Variables de estudio

\begin{tabular}{|c|c|c|}
\hline VARIABLES DE E & VARIABLES DE PROCESO & VARIABLES DE PRODUCTO \\
\hline 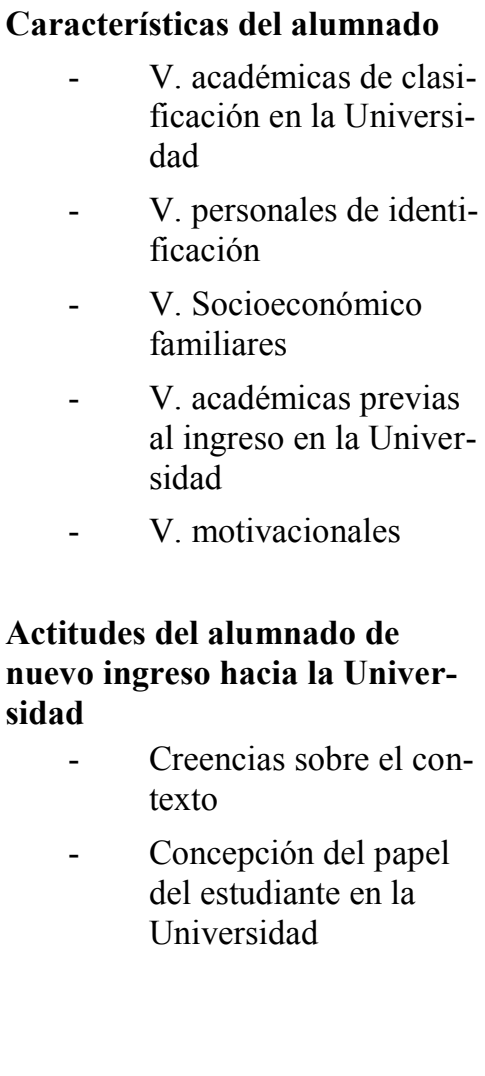 & 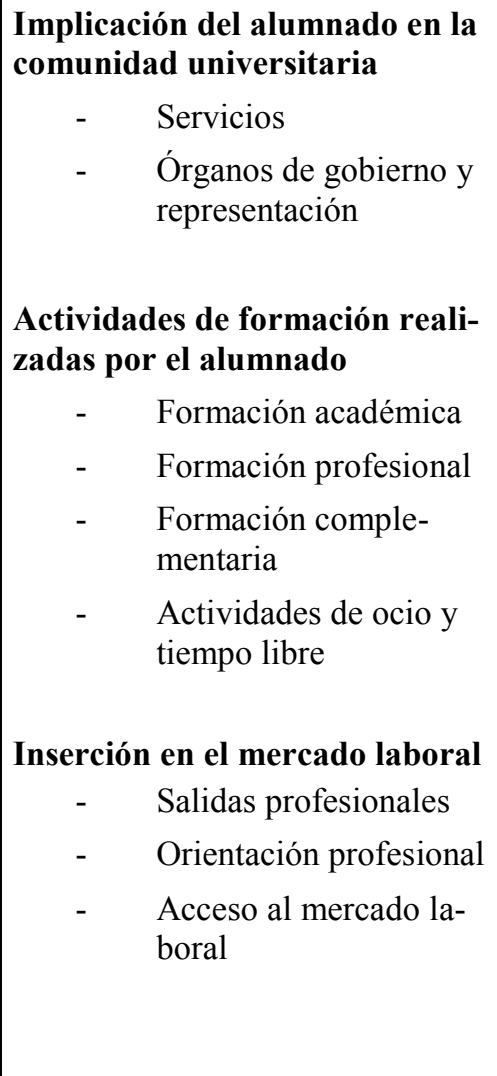 & 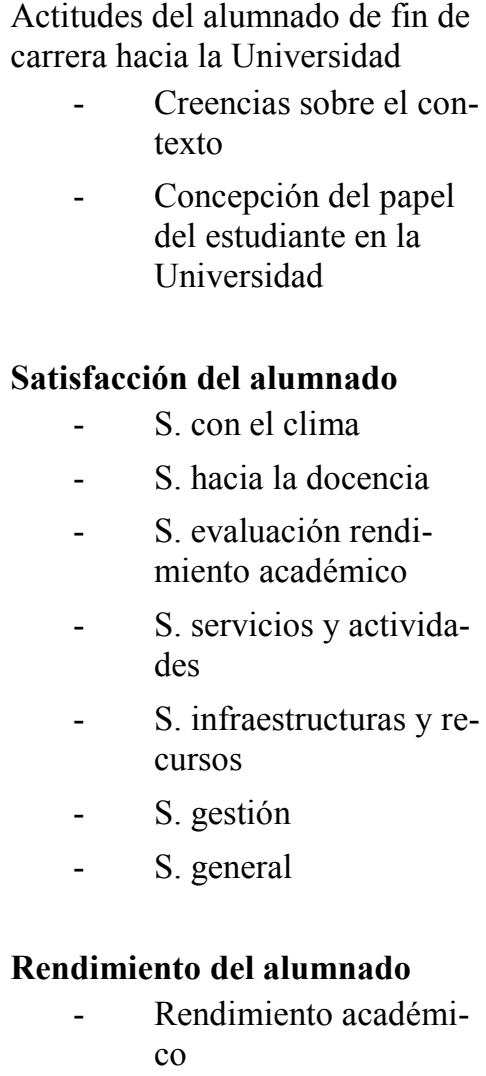 \\
\hline
\end{tabular}

En segundo lugar, las variables de proceso hacen referencia al funcionamiento interno de la Universidad y al grado en el que el alumno participa de ese funcionamiento, así como los elementos relacionados con su formación, dando cuenta de las actividades realizadas y los mecanismos de inserción laboral llevados a cabo.

Por último, las variables de producto abarcan, por un lado, las actitudes del alumnado de último año de carrera con respecto a lo que ha sido su paso por la Universidad. En un segundo momento, se han tratado los elementos relacionados con su satisfacción respecto de la institución universitaria. En tercer lugar se analizan las características de su rendimiento académico y, en cuarto lugar, se ha tratado de obtener información que sirva para definir una Universidad de calidad.

Con la ayuda del programa informático NUDIST 4.0, lo que tratamos de realizar con esta información fue explorar, describir y analizar patrones culturales y sociales dentro de la vida cotidiana universitaria. De este modo, podremos identificar sus características básicas e inferir particularidades con respecto al sistema que la Universidad representa. 


\section{Instrumentos}

La obtención de información referente a este conjunto de variables se llevó a cabo gracias a dos instrumentos bien diferenciados. En primer lugar, se diseñó un cuestionario dirigido a los alumnos de primeros y últimos cursos, dado que son la principal fuente de información. Estaba compuesto por 136 preguntas de las que 125 fueron cerradas (ítems de valoración del grado de acuerdo o desacuerdo con una determinada afirmación, a lo largo de una escala de cinco puntos), 4 semicerradas y 7 abiertas, estructuradas a lo largo de diez dimensiones en las cuales se concretaban todas las variables del estudio. Su cumplimentación aportó un total de 103672 respuestas, de las que 101828 fueron sometidas a análisis cuantitativo (estudios descriptivo, inferencial, correlacional y multivariante) y 1844 a análisis cualitativo (análisis de contenido). En segundo lugar, trabajamos con datos oficiales referente a variables de identificación y rendimiento académico principalmente.

Debido a la imposibilidad de esperar cinco o seis años a que los estudiantes encuestados concluyeran sus estudios, llevamos a cabo un procedimiento transversal donde los datos de entrada fueron recogidos del alumnado de nuevo ingreso y los datos de salida de los estudiantes de último curso de carrera.

\section{Resultados del análisis cualitativo}

\section{Análisis cualitativo}

Del total de respuestas abiertas obtenidas (1844), seleccionamos las 1708 correspondientes a los cuatro últimos ítems del cuestionario, principales referentes del objetivo propuesto. Éstas fueron analizadas a través de la técnica del análisis de contenido, que responde al carácter que tiene el ser humano de indagación y descubrimiento, el más allá de la información textual, por lo que se centra en buscar dentro de las distintas locuciones humanas aquellas particularidades del lenguaje que aporten significados intersubjetivos característicos de sus sistemas de comunicación. Se trata de obtener registros de ese contenido para inferir una serie de conclusiones que responden a una serie de hipótesis preestablecidas y unas teorías de partida (Bartolomé, 1990, p. 15). 
Tal y como reflejan los datos de la tabla 1, la pregunta que fue respondida con mayor asiduidad ha sido la número 132 (definición de Universidad de calidad), contestada por el 61.21\% de los encuestados, seguida de la número 134 (aspectos negativos de la formación universitaria), respondida por el $54.52 \%$ de los alumnos. En definitiva, la proporción media de respuesta ha sido del $52.92 \%$.

Tabla 1: Preguntas abiertas, respuestas válidas y proporciones de respuesta válidas sometidas a análisis de contenido

\begin{tabular}{|c|l|l|c|c||}
\hline $\begin{array}{c}\text { No pre- } \\
\text { gunta }\end{array}$ & $\begin{array}{c}\text { Nombre } \\
\text { variable }\end{array}$ & \multicolumn{1}{|c|}{ Pregunta } & $\begin{array}{c}\text { Respuestas } \\
\text { válidas }\end{array}$ & $\begin{array}{c}\text { Proporción } \\
\text { de respuesta }\end{array}$ \\
\hline \hline 132 & UNICALI & $\begin{array}{l}\text { ¿Qué es para ti una Universidad de } \\
\text { calidad? }\end{array}$ & 494 & $61.21 \%$ \\
\hline 133 & FORMAPOS & $\begin{array}{l}\text { ¿Qué aspectos consideras más positivos } \\
\text { en tu formación universitaria? }\end{array}$ & 387 & $47.95 \%$ \\
\hline 134 & FORMANE & $\begin{array}{l}\text { ¿Qué aspectos consideras más negati- } \\
\text { vos en tu formación universitaria? }\end{array}$ & 440 & $54.52 \%$ \\
\hline 135 & PROPFORM & $\begin{array}{l}\text { ¿Qué propondrías para mejorar tu for- } \\
\text { mación universitaria? }\end{array}$ & 387 & $47.95 \%$ \\
\hline
\end{tabular}

El sistema de categorías base para la realización del análisis está formado por un árbol con cuatro ramas, correspondientes a cada una de las preguntas del cuestionario que son objeto de este estudio. La codificación se realizó a posteriori una vez recogidas las respuestas, lo que ha supuesto un primer acercamiento al análisis de los resultados. El número total de categorías propuestas ha sido de 334, una vez asignados los documentos a cada una de las categorías de análisis, el proceso ha de continuar con la disposición de los datos, la obtención de resultados y la verificación de conclusiones.

\section{Disposición y transformación de los datos}

Dada la gran cantidad de información textual que es objeto de análisis y el elevado número de categorías, los resultados obtenidos son muy extensos, por lo que, un primer paso en el análisis de los mismos será la disposición de datos. Se trata de un procedimiento de reordenación que permite presentarlos de manera abarcable y operativa de cara a resolver las cuestiones de la investigación (Rodríguez et al., 1995: 255).

En nuestro caso, y atendiendo al trabajo realizado por Sánchez, Prado y Martín (2001) sobre el análisis cualitativo de los temas transversales implícitos en libros de lecturas, hemos 
utilizado la matriz de la función coding para cada una de las cuatro principales categorías de análisis (aspectos positivos de la formación universitaria, aspectos negativos de la formación universitaria, propuestas para mejorar la formación universitaria y definición de Universidad de calidad) que determina la existencia (1) o no (0) de dichas unidades textuales en las respuestas analizadas y cuáles han sido más recurrentes. Dada su extensión no se pueden reflejar en este trabajo.

\section{Obtención y verificación de conclusiones}

En este momento, surge la necesidad de llevar a cabo un estudio pormenorizado de la información de modo que nos ofrezca conclusiones significativas para responder a los objetivos del estudio.

Para tal fin, hemos llevado a cabo un triple análisis. En primer lugar, realizamos un estudio del porcentaje de contenido textual de cada una de las respuestas abiertas (make report); en segundo lugar, analizamos los resultados obtenidos tras la conexión entre códigos (union) $\mathrm{y}$, por último, efectuamos una jerarquización de los mismos a través del empleo de diferentes operadores booleanos (collect).

Los principales datos obtenidos son:

- Una buena formación universitaria ha de estar caracterizada por un componente personal y profesional que permita al alumnado desarrollarse socialmente a través de la adquisición de habilidades, actitudes y valores. Una buena formación es aquella que potencia las relaciones interpersonales y oferta prácticas adecuadas a los objetivos formativos propuestos.

- Los males que afectan a la formación de los alumnos son, principalmente, planes de estudios sobrecargados de asignaturas, muchas de ellas innecesarias y un exceso de contenidos teóricos; falta de atención por parte del profesorado; escasez de prácticas profesionales; sobrecarga de horarios; masificación; falta de información; ausencia de un sistema de orientación profesional; competitividad y desmotivación de los alumnos; metodología didáctica basada en clases magistrales; evaluación cuantitativa y recursos materiales inadecuados e insuficientes. 
- Las propuestas planteadas para evitar los males anteriores se centran, fundamentalmente, en la mejora del sistema de prácticas, en la necesidad de orientar laboralmente a los alumnos, en la renovación de los planes de estudios, en la introducción de nuevas metodologías didácticas, nuevas ideas referentes al profesorado (cualificación y atención al alumno) y al alumnado (relaciones y participación), en nuevos sistemas de organización administrativa de las diferentes titulaciones y en una mejora de los recursos materiales para el correcto desarrollo de las actividades propuestas.

- En definitiva y a partir de las aportaciones de los estudiantes que han compuesto la muestra de estudio, podemos considerar que una Universidad de calidad es aquélla que aporta una formación integral al alumno, de modo que responda a sus necesidades laborales y sociales. Es aquélla que posea un profesorado cualificado y satisfecho, unos recursos materiales adecuados a las necesidades de la comunidad universitaria, y que cumpla sus objetivos. Es de calidad una Universidad que fomente la investigación y, en menor medida, aquélla que posea una gran diversidad de titulaciones, servicios adicionales y atienda a los estudiantes de forma individualizada.

\section{Resultados del análisis cuantitativo}

Las manifestaciones hasta aquí planteadas se han visto validadas a través de un estudio de regresión múltiple y un análisis discriminante, técnicas que nos han ayudado a explicar las relaciones entre las distintas variables y su grado de interdependencia, así como a especificar aquellos elementos que definen, desde la opinión del alumnado, una institución universitaria de calidad.

\section{Análisis de regresión múltiple}

El objetivo de este análisis se centra en cuantificar la relación entre la variable dependiente y las variables independientes, y establecer con qué grado de confianza podemos afirmar que la cuantificación observada se ajusta a la realidad (Guillén, 1992, p. 8). 
El primer paso consistió en seleccionar las variables que fueran relevantes para los objetivos del estudio. En nuestro caso, y a partir de los conclusiones obtenidas en el estudio cualitativo de respuestas abiertas, hemos identificado operativamente una serie de variables que conforman un modelo de relación y que van siendo introducidas en función de la conexión existente entre el porcentaje de unidades textuales indexadas en la categoría especificada y el ítem del cuestionario al que hacen referencia (ver tabla 2).

Tabla 2: Variables en el modelo de regresión múltiple

\begin{tabular}{|c|c|c|c|}
\hline $\begin{array}{l}\text { Tipo de } \\
\text { variable }\end{array}$ & $\begin{array}{l}\text { Resultados del estudio cualita- } \\
\text { tivo }\end{array}$ & Variable & Coincidencia con el ítem... \\
\hline Criterio & $\begin{array}{l}\text { Definición de Universidad de } \\
\text { calidad }\end{array}$ & $\begin{array}{l}\text { Y: Universidad de cali- } \\
\text { dad }\end{array}$ & $\begin{array}{l}\text { 103: La enseñanza que recibo es de } \\
\text { calidad }\end{array}$ \\
\hline \multirow[t]{13}{*}{ Predictoras } & $\begin{array}{l}\text { Formación integral del alumnado, } \\
\text { donde cobran importancia los } \\
\text { componentes profesionales y } \\
\text { personales }\end{array}$ & $\mathrm{X}_{1}$ : Formación integral & $\begin{array}{l}\text { 58: Mi paso por la Universidad me está } \\
\text { formando, no sólo como un profesional }\end{array}$ \\
\hline & $\begin{array}{l}\text { Oferta de formación teórica y } \\
\text { práctica importante }\end{array}$ & $\begin{array}{l}\mathrm{X}_{2}: \text { Formación multidi- } \\
\text { mensional }\end{array}$ & $\begin{array}{l}\text { 69: Los profesores integran teoría y } \\
\text { práctica en sus asignaturas }\end{array}$ \\
\hline & $\begin{array}{l}\text { Instalaciones y recursos adecua- } \\
\text { dos a las necesidades de la comu- } \\
\text { nidad universitaria }\end{array}$ & $\begin{array}{l}\mathrm{X}_{3}: \text { Instalaciones y } \\
\text { recursos }\end{array}$ & $\begin{array}{l}\text { 97: Las instalaciones se adecúan a mis } \\
\text { necesidades }\end{array}$ \\
\hline & $\begin{array}{l}\text { Orientación laboral en las diferen- } \\
\text { tes titulaciones }\end{array}$ & $\mathrm{X}_{4}$ : Orientación laboral & $\begin{array}{l}\text { 122: A lo largo de mi formación he } \\
\text { recibido información sobre el mundo } \\
\text { del trabajo y las salidas profesionales de } \\
\text { mi titulación }\end{array}$ \\
\hline & $\begin{array}{l}\text { Profesorado motivador, motivado } \\
\text { y satisfecho }\end{array}$ & $\mathrm{X}_{5}$ : Profesorado & $\begin{array}{l}\text { 91: Me siendo bien atendido por mis } \\
\text { profesores }\end{array}$ \\
\hline & $\begin{array}{l}\text { Alumnos satisfechos con la for- } \\
\text { mación recibida }\end{array}$ & $\begin{array}{l}\mathrm{X}_{6}: \text { Satisfacción del } \\
\text { alumnado }\end{array}$ & $\begin{array}{l}\text { 92: Mis expectativas son respecto a la } \\
\text { formación que estoy recibiendo se están } \\
\text { cumpliendo satisfactoriamente }\end{array}$ \\
\hline & $\begin{array}{l}\text { Transmisión de valores como la } \\
\text { libertad y formación de actitudes } \\
\text { entre las que destaca el espíritu } \\
\text { crítico }\end{array}$ & $\begin{array}{l}X_{7}: \text { Formación en valo- } \\
\text { res y actitudes }\end{array}$ & $\begin{array}{l}\text { 72: Estoy adquiriendo normas, actitudes } \\
\text { y cualidades personales específicas de } \\
\text { la profesión }\end{array}$ \\
\hline & Formación en habilidades & $\begin{array}{l}\mathrm{X}_{8}: \text { Formación en habi- } \\
\text { lidades }\end{array}$ & $\begin{array}{l}\text { 63: Estoy adquiriendo la habilidad de } \\
\text { reflexionar y aprender }\end{array}$ \\
\hline & $\begin{array}{l}\text { Evaluación cuantitativa y cualita- } \\
\text { tiva }\end{array}$ & $\begin{array}{l}\mathrm{X}_{9} \text { : Evaluación del } \\
\text { alumnado }\end{array}$ & $\begin{array}{l}\text { 94: La evaluación responde a todos los } \\
\text { aspectos de mi formación }\end{array}$ \\
\hline & $\begin{array}{l}\text { Atención individualizada a los } \\
\text { alumnos }\end{array}$ & $\mathrm{X}_{10}:$ Sistema de tutorías & $\begin{array}{l}\text { 29: Ser asistidos mediante un buen } \\
\text { sistema de tutorías }\end{array}$ \\
\hline & $\begin{array}{l}\text { Servicios adicionales a disposi- } \\
\text { ción de la comunidad universita- } \\
\text { ria }\end{array}$ & $\begin{array}{l}\mathrm{X}_{11}: \text { Servicios y activi- } \\
\text { dades }\end{array}$ & $\begin{array}{l}\text { 96: Los servicios y actividades oferta- } \\
\text { dos por la Universidad responden a mis } \\
\text { necesidades }\end{array}$ \\
\hline & $\begin{array}{l}\text { Ofrecer Información al alumnado } \\
\text { sobre la institución }\end{array}$ & $\begin{array}{l}\mathrm{X}_{12}: \text { Información sobre } \\
\text { el funcionamiento de la } \\
\text { Universidad }\end{array}$ & $\begin{array}{l}\text { 37: Recibir una adecuada información } \\
\text { sobre sus derechos como estudiante, así } \\
\text { como del funcionamiento general de la } \\
\text { Universidad }\end{array}$ \\
\hline & $\begin{array}{l}\text { Cumplir con los objetivos ini- } \\
\text { cialmente propuestos }\end{array}$ & $\begin{array}{l}\mathrm{X}_{13}: \text { Objetivos de la } \\
\text { institución }\end{array}$ & $\begin{array}{l}\text { 42: Cooperar para la consecución de los } \\
\text { objetivos de la institución }\end{array}$ \\
\hline
\end{tabular}


La variable criterio hace referencia, en el estudio cualitativo, a la definición de Universidad de calidad, identificada en el conjunto de variables como el ítem número 103 del cuestionario, y el grupo de variables predictoras está formado por aquellas que hacen referencia a la formación integral y multidimensional, instalaciones, orientación laboral, profesorado, satisfacción del alumnado, formación en actitudes y valores, adquisición de habilidades, tutorías, servicios, actividades, información y gestión.

Tras la descripción realizada, el propósito planteado es mostrar, a partir de la capacidad de predicción sobre la variable criterio y eligiendo aquel modelo más parsimonioso, en orden de mayor a menor importancia, las dimensiones alrededor de las cuales gira el concepto de calidad universitaria. El modelo de regresión que se presenta queda reflejado en la figura 2.

Figura 2: Modelo de regresión múltiple

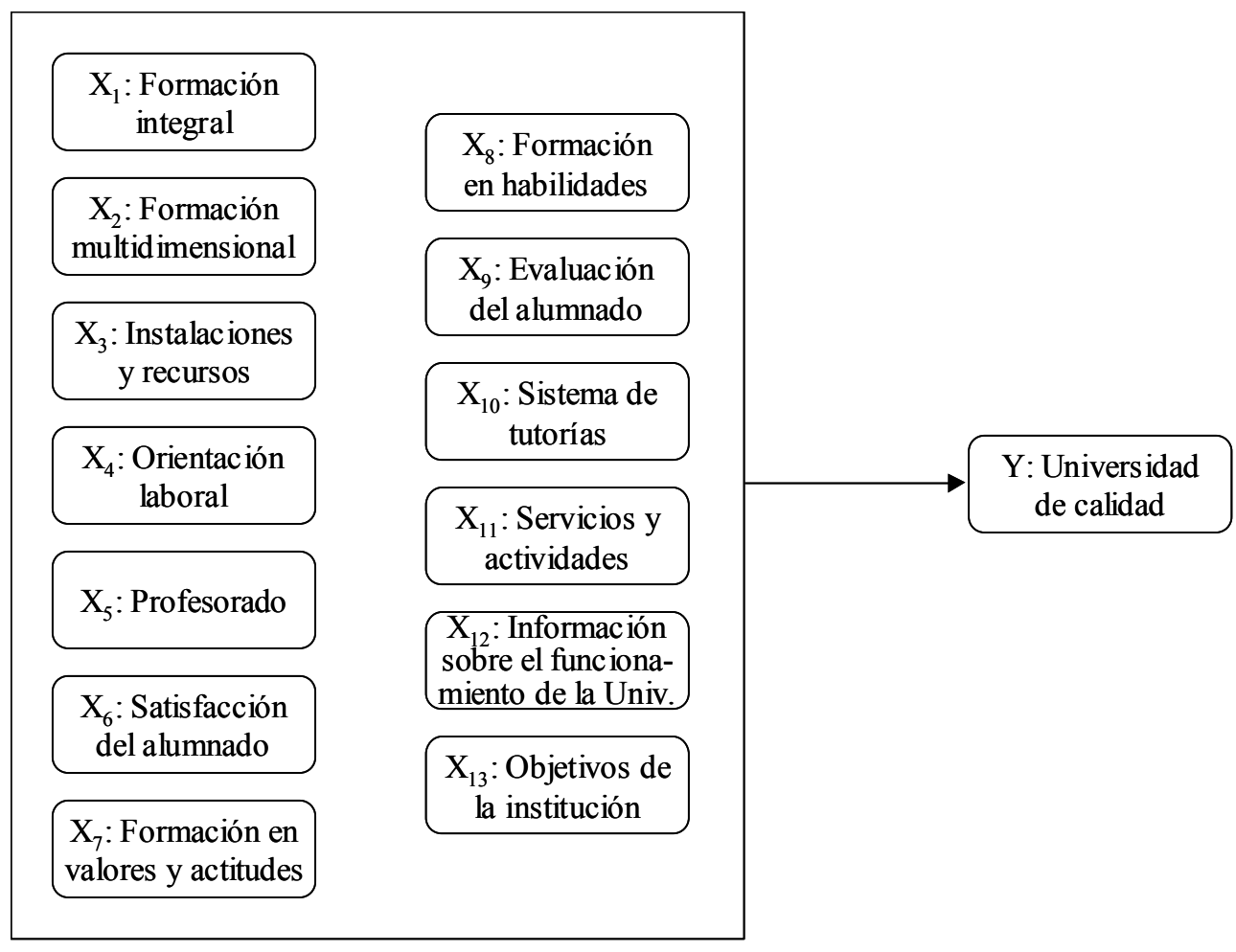

Una vez hemos especificado las variables que van a ser utilizadas para explicar la variable dependiente y el orden en el que éstas van a ser introducidas, nos decantamos por utilizar el método de inclusión stepwise o pasos sucesivos que, tal y como destaca Etxeberría (1999: 89) se trata del método más completo y el que aporta más información. A partir de la inclusión de la segunda variable, en cada etapa se va analizando la significatividad de todas las variables que en ese momento están incluidas en la ecuación, de tal modo que, si alguna de 
ellas no aporta información, es eliminada del modelo. En definitiva, el modelo de regresión múltiple queda planteado del siguiente modo (ver tabla 3).

Tabla 3: Resumen del modelo de regresión múltiple

\begin{tabular}{|c|c|l|c|c|c|c|c|}
\hline Pasos & $\begin{array}{c}\text { Variable } \\
\text { criterio }\end{array}$ & \multicolumn{1}{|c|}{ Variables predictoras } & $\mathbf{R}$ & $\mathbf{R}^{2}$ & Delta $\mathbf{R}$ & $\mathbf{F}$ & $\mathbf{p}$ \\
\hline \hline 1 & $\mathrm{Y}$ & $\mathrm{X}_{6}$ & 0.574 & 0.330 & 0.330 & 363.508 & 0.000 \\
2 & $\mathrm{Y}$ & $\mathrm{X}_{6}, \mathrm{X}_{5}$ & 0.622 & 0.387 & 0.057 & 232.775 & 0.000 \\
3 & $\mathrm{Y}$ & $\mathrm{X}_{6}, \mathrm{X}_{5}, \mathrm{X}_{3}$ & 0.643 & 0.413 & 0.026 & 172.486 & 0.000 \\
4 & $\mathrm{Y}$ & $\mathrm{X}_{6}, \mathrm{X}_{5}, \mathrm{X}_{3}, \mathrm{X}_{1}$ & 0.656 & 0.430 & 0.017 & 138.718 & 0.000 \\
5 & $\mathrm{Y}$ & $\mathrm{X}_{6}, \mathrm{X}_{5}, \mathrm{X}_{3}, \mathrm{X}_{1}, \mathrm{X}_{2}$ & 0.662 & 0.438 & 0.008 & 114.526 & 0.000 \\
6 & $\mathrm{Y}$ & $\mathrm{X}_{6}, \mathrm{X}_{5}, \mathrm{X}_{3}, \mathrm{X}_{1}, \mathrm{X}_{2}, \mathrm{X}_{4}$ & 0.666 & 0.444 & 0.006 & 97.644 & 0.000 \\
7 & $\mathrm{Y}$ & $\mathrm{X}_{6}, \mathrm{X}_{5}, \mathrm{X}_{3}, \mathrm{X}_{1}, \mathrm{X}_{2}, \mathrm{X}_{4}, \mathrm{X}_{9}$ & 0.670 & 0.448 & 0.004 & 85.019 & 0.000 \\
8 & $\mathrm{Y}$ & $\mathrm{X}_{6}, \mathrm{X}_{5}, \mathrm{X}_{3}, \mathrm{X}_{1}, \mathrm{X}_{2}, \mathrm{X}_{4}, \mathrm{X}_{9}, \mathrm{X}_{7}$ & 0.672 & 0.452 & 0.004 & 75.303 & 0.000 \\
\hline
\end{tabular}

Los resultados obtenidos resaltan que, de las trece variables predictoras incorporadas, deducidas de las respuestas abiertas que el alumno aportó en la cuestión sobre su definición de Universidad de calidad, sólo han resultado seleccionadas ocho variables, con una explicación total de la varianza del criterio del 45.2\%. El gráfico 3 representa la probabilidad normal que guía nuestro modelo. Como puede apreciarse, los valores muestrales se superponen prácticamente a la diagonal principal, indicando su proximidad casi absoluta a la normalidad.

Figura 3: Gráfico de probabilidad

Variable dependiente: Universidad de calidad

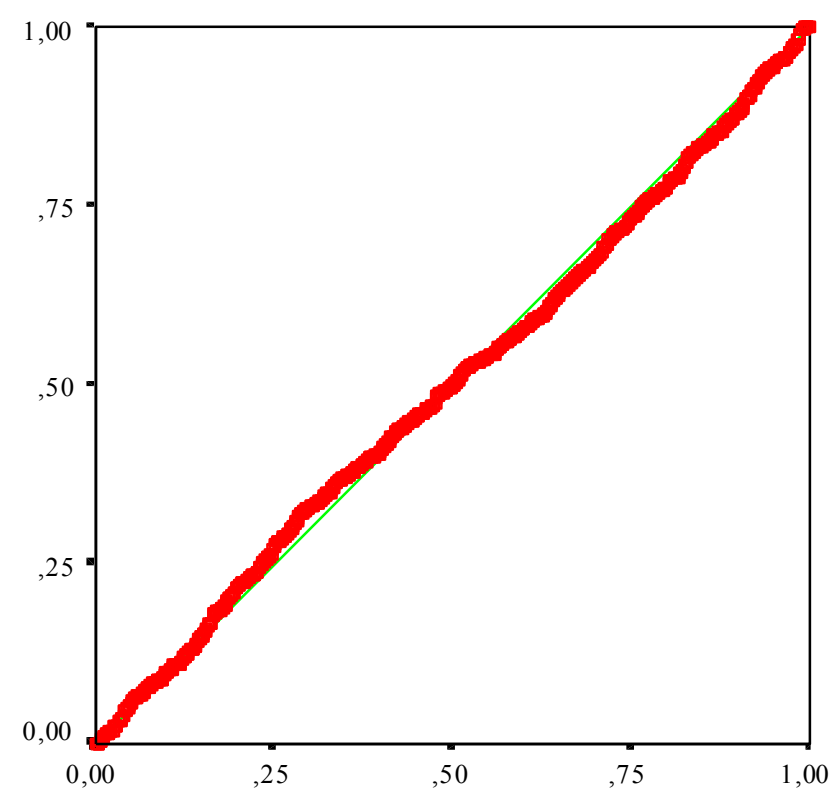

Prob. acum observada 
El orden de incorporación al modelo de las variables ha sido el siguiente:

$1^{\circ}$. Satisfacción del alumnado $\left(\mathrm{X}_{6}\right)$, con una explicación de la variabilidad del criterio del 33\%. Es decir, los resultados vienen a confirmar la importancia que en el listado de indicadores de producto de una institución se le concede a la "satisfacción de los usuarios". Según este trabajo, la variable que correlaciona de un modo más intenso cuantitativamente y, por tanto, predice una opinión positiva hacia el recibir una "enseñanza de calidad" está relacionada con el nivel de satisfacción hacia la formación que se está recibiendo. Si se quiere, estamos definiendo otra vez el concepto tan acuñado de "calidad". El modelo de evaluación diseñado por Malcolm Baldrige tiene como principal aportación la necesidad de responder a las necesidades y expectativas de los clientes (CIGAL, 2000), elemento que ha configurado los principios de la calidad total aplicados a la educación superior. En este sentido, hemos tenido que recordar que la satisfacción de los estudiantes es la dimensión que más peso tiene en las políticas de evaluación de la calidad de los países europeos, incluida España (Consejo de Universidades, 1998).

$2^{\circ}$. Profesorado $\left(\mathrm{X}_{5}\right)$, con una explicación de la variabilidad del criterio del 5.7\%. En este sentido, la siguiente variable incorporada reduce bastante la explicación de la variabilidad $\mathrm{y}$, por tanto, podemos decir que prácticamente la explicación de lo que el alumno entiende por una enseñanza de calidad se explica por la variable satisfacción. Sin embargo, es curioso observar que tras esta variable aparezca la atención del profesorado, o el hecho de sentirse atendido, tratado individualmente como persona. Este resultado induce a pensar que, desde el punto de vista del alumnado, una atención más individualizada constituye un elemento sustantivo a la hora de otorgar mérito a la enseñanza. Recordemos esta dimensión en la evaluación docente y el peso que aquí se le otorga (Grupo Helmántica, 1995).

$3^{\circ}$. Instalaciones y recursos $\left(\mathrm{X}_{3}\right)$, con una explicación de la variabilidad del criterio del $2.6 \%$. Variable que reduce aun más la explicación de la variabilidad del criterio (calidad). Sin embargo, también es interesante la reflexión a realizar a partir de la entrada de esta variable relacionada con el ámbito de las infraestructuras en la docencia universitaria. Actualmente, donde se están planteando desde la gestión académica ciertos cambios motivados por la incorporación a la sociedad de la información y el conocimiento de las nuevas tecnologías, observamos cómo desde el usuario alumno se realiza una llamada de atención hacia este aspecto. 
Podemos decir que el alumno sí relaciona "calidad" de la enseñanza con el grado en el que las “instalaciones" responden a sus actuales necesidades.

$4^{\mathrm{o}}$. Formación integral $\left(\mathrm{X}_{1}\right)$, con una explicación de la variabilidad del criterio del $1.7 \%$. A pesar de esta aportación, el alumno es consciente de que una Universidad de calidad ha de aportar un conjunto de conocimientos que le sirvan para enfrentarse al mundo en el que se instale una vez haya concluido sus estudios. Es interesante cómo defienden que la formación universitaria ha de dotarles de los conocimientos y los recursos necesarios para desenvolverse profesional y personalmente en la vida adulta.

$5^{\circ}$. Formación multidimensional $\left(\mathrm{X}_{2}\right)$, con una explicación de la variabilidad del criterio del $0.8 \%$. Esta mínima aportación viene a decir que los estudiantes consideran que es de calidad aquella Universidad que aporte una formación teórica y práctica en las diferentes titulaciones que oferte, a través de diferentes actividades docentes.

$6^{\circ}$. Orientación laboral $\left(\mathrm{X}_{4}\right)$, con una explicación de la variabilidad del criterio del $0.6 \%$. A pesar de reducirse aún más esta explicación, los estudiantes consideran que la institución universitaria ha de aportar información sobre el mundo del trabajo, así como de las salidas profesionales de cada titulación. Asociado a la formación integral, una Universidad de calidad se caracteriza por dotar de un componente laboral a la formación en el que tenga especial relevancia la orientación para el acceso al empleo.

$7^{\circ}$. Evaluación del alumnado $\left(\mathrm{X}_{9}\right)$, con una explicación de la variabilidad del criterio del $0.4 \%$. Esta variable explica una casi ausente explicación al modelo de regresión. Sin embargo, parece que los alumnos quieren destacar que un aspecto a valorar en la definición de la Universidad de calidad son los sistemas de evaluación de su rendimiento. Identifican un factor de calidad la adecuación de la evaluación a los diferentes aspectos de la formación: teóricos, prácticos, conocimientos, experiencias, participación, etc.

$8^{\circ}$. Formación en valores y actitudes $\left(\mathrm{X}_{7}\right)$, con una explicación de la variabilidad del criterio del $0.4 \%$. Se trata de la última variable que entra a formar parte del modelo y cuya aportación es casi nula. Sin embargo, hay que destacar que para los estudiantes es relevante una formación basada en la adquisición de normas, actitudes y cualidades, necesarias para el desenvolvimiento personal, profesional y social. 
Las variables eliminadas en este modelo han sido: formación en habilidades $\left(\mathrm{X}_{8}\right)$, sistema de tutorías $\left(\mathrm{X}_{10}\right)$, servicios y actividades $\left(\mathrm{X}_{11}\right)$ información sobre el funcionamiento de la Universidad $\left(\mathrm{X}_{12}\right)$ y objetivos de la institución $\left(\mathrm{X}_{13}\right)$. Considerados como elementos definitorios de una institución de calidad, su aportación al modelo de regresión múltiple no ha sido significativa.

\section{Análisis discriminante}

Esta técnica se utilizó con la finalidad de disminuir la posibilidad de obtener resultados significativos simplemente por azar, tratando de descubrir aquellos elementos capaces de describir las diferencias entre los alumnos que consideran que la institución en la que cursan sus estudios es o no de calidad.

Tomando como referencia la variable criterio del modelo de regresión múltiple (Universidad de calidad) codificada de 1 a 5 (totalmente en desacuerdo a totalmente de acuerdo), los grupos de discriminación son:

1. Grupo calidad baja: formado por todos aquellos estudiantes que respondieron 1 y 2 en el ítem de referencia.

2. Grupo calidad media (missing): formado por todos los alumnos que puntuaron con el valor 3 el ítem.

3. Grupo calidad alta: formado por todos los estudiantes que respondieron 4 y 5 en el ítem.

A continuación, seleccionamos las variables que definen el modelo, que son los predictores seleccionados en el estudio de regresión múltiple (13 variables), procediendo al análisis especificado.

Siguiendo la medida de discriminación Lambda de Wilks, de entre las técnicas stepwise existentes, el modelo ha eliminado siete variables, de modo que son seis las que son capaces de discriminar a los estudiantes en los dos grupos señalados como alto y bajo (ver tabla 4). 
Tabla 4: Variables introducidas en cada paso y valores Lambda de Wilks conseguidos

\begin{tabular}{|c|l|c|c|c|}
\hline Pasos & \multicolumn{1}{|c|}{ Variables } & $\begin{array}{r}\text { Lambda } \\
\text { de Wilks }\end{array}$ & F & p \\
\hline \hline 1 & Satisfacción del alumnado & 0.611 & 307.817 & 0.000 \\
2 & Profesorado & 0.558 & 190.638 & 0.000 \\
3 & Instalaciones y recursos & 0.536 & 138.917 & 0.000 \\
4 & Formación multidimensional & 0.521 & 110.183 & 0.000 \\
5 & Formación integral & 0.511 & 91.660 & 0.000 \\
6 & Formación en valores y actitudes & 0.505 & 78.117 & 0.000 \\
\hline
\end{tabular}

A partir de estas variables se ha construido la función discriminante, que nos ha permitido interpretar el poder de discriminación de las variables introducidas. Hemos encontrado un valor Lambda para la función de 0.505 , adoptando ji cuadrado un valor de 328.018, que resulta significativo a un nivel de significación de 0.01. En consecuencia, podemos decir que esta función da lugar a diferencias significativas entre los grupos. Tomando como referencia la correlación intragrupo de cada una de las variables con la función discriminante (coeficientes de estructura), tal y como muestra la tabla 5, se especifica la contribución a la discriminación de los dos grupos de la variable criterio de cada una de las variables.

Tabla 5: Coeficientes de estructura de la función discriminante

\begin{tabular}{||l|c||}
\hline \multicolumn{1}{|c|}{ Variables } & $\begin{array}{c}\text { Coeficientes } \\
\text { de estructura }\end{array}$ \\
\hline Satisfacción del alumnado & 0.806 \\
Profesorado & 0.664 \\
Formación en valores y actitudes & 0.500 \\
Instalaciones y recursos & 0.494 \\
Formación multidimensional & 0.465 \\
Formación integral & 0.422 \\
\hline
\end{tabular}

De este modo se aprecia, al igual que en el estudio anterior, que la variable que contribuye a definir, en primer lugar a ambos grupos, es la satisfacción del alumnado, en el sentido de ver atendidas sus necesidades y cumplidas sus expectativas. En segundo lugar, el profesorado ha de atender de forma satisfactoria a los estudiantes, a los que se ha de transmitir una formación en la que destaquen valores como la libertad y actitudes entre las que predomine el espíritu crítico. Por su parte, una Universidad de calidad ha de poseer unas instalaciones y unos recursos adecuados a las necesidades de la comunidad universitaria. Esta institución ha de destacar por la oferta de una importante formación que integre la teoría y la práctica, así 
como de una formación integral donde cobren importancia los componentes profesionales y personales.

Repetido este estudio clasificando a los alumnos encuestados en función de su rendimiento académico (ver tabla 6), aquellos con bajo rendimiento consideran que una Universidad de calidad es aquella que satisfaga al alumnado, cuyo profesorado sea atento con los estudiantes, donde estos sean evaluados desde una doble perspectiva cuantitativa y cualitativa, que ofrezca una formación integral y que, para ello, posea las instalaciones y los recursos adecuados.

Tabla 6: Coeficientes de estructura de la función discriminante en función del rendimiento académico

\begin{tabular}{|c|c|c|}
\hline & Variables & $\begin{array}{l}\text { Coeficientes } \\
\text { de estructura }\end{array}$ \\
\hline \multirow{5}{*}{$\begin{array}{l}\text { Alumnos con bajo ren- } \\
\text { dimiento académico }\end{array}$} & Satisfacción del alumnado & 0.776 \\
\hline & Profesorado & 0.624 \\
\hline & Evaluación del alumnado & 0.584 \\
\hline & Formación integral & 0.444 \\
\hline & Instalaciones y recursos & 0.435 \\
\hline \multirow{2}{*}{$\begin{array}{l}\text { Alumnos con alto ren- } \\
\text { dimiento académico }\end{array}$} & Formación en valores y actitudes & 0.606 \\
\hline & Formación en habilidades & 0.515 \\
\hline
\end{tabular}

Por su parte, los elementos característicos que definen una Universidad de calidad para los alumnos que poseen un alto rendimiento académico son una formación cuya base sea la transmisión de valores y actitudes y habilidades como la reflexión y el aprendizaje.

Realizado de nuevo este análisis atendiendo a la rama de especialización a la que pertenecen los estudios en los que se encuentran matriculados los estudiantes, encontramos los siguientes resultados (ver tabla 7):

- Ciencias de la Salud: los estudiantes de estas especialidades consideran que es de calidad una Universidad donde su profesorado atienda satisfactoriamente al alumnado, que oferte una formación integral (profesional y personal), así como una formación que combine la teoría y la práctica. 
Tabla 7: Coeficientes de estructura de la función discriminante en función de la rama de especialización

\begin{tabular}{|l||l|c|}
\cline { 2 - 3 } \multicolumn{1}{l|}{} & \multicolumn{1}{|c|}{ Variables } & $\begin{array}{c}\text { Coeficientes } \\
\text { de estructura }\end{array}$ \\
\hline \multirow{3}{*}{ Ciencias de la Salud } & Frofesorado & 0.801 \\
& Formación integral & 0.551 \\
& Formación multidimensional & 0.536 \\
\hline \multirow{3}{*}{ Humanidades } & Evaluación del alumnado & 0.648 \\
& Formación integral & 0.518 \\
\hline \multirow{3}{*}{ Jurídico-Sociales } & Satisfacción del alumnado & 0.363 \\
& Formación en valores y actitudes & 0.207 \\
\hline \multirow{2}{*}{ Técnicas } & Frofesorado & 0.914 \\
& Formación integral & 0.455 \\
\hline & Frofesorado & 0.915 \\
& Formación en valores y actitudes & 0.589 \\
& Instalaciones y recursos & 0.375 \\
\hline & Orientación laboral & 0.585 \\
& Profesorado & 0.569 \\
\hline
\end{tabular}

- Experimentales: estos alumnos manifiestan que una correcta evaluación de sus aprendizajes, tanto cualitativa como cuantitativa, es el elemento que mejor caracteriza una Universidad de calidad. Asimismo, ha de ofrecer una formación integral, atender a las necesidades y expectativas de sus estudiantes y aportar valores y actitudes en las diferentes actividades formativas.

- Humanidades: estos estudiantes, al igual que sus compañeros de Ciencias de la Salud, consideran que el elemento que mejor define una Universidad de calidad es que posea un profesorado que les atienda de forma satisfactoria. Por otro lado, ha de ofrecer una formación integral, caracterizada por elementos profesionales y personales.

- Jurídico-Sociales: un profesorado que atienda al alumnado, una formación que ayude a transmitir valores y actitudes y la posesión de instalaciones y recursos adecuados a las necesidades de la comunidad, son los factores que, para este grupo de alumnos, conforman una Universidad de calidad. 
- Técnicas: los estudiantes pertenecientes a estas titulaciones manifiestan que los alumnos han de recibir información sobre el mundo laboral y las salidas profesionales de sus estudios. En segundo lugar, el profesorado ha de atender a este alumnado de forma satisfactoria. En tercer lugar, la institución ha de poseer unas instalaciones y unos recursos adecuados a las diferentes actividades y, por último, la evaluación (cuantitativa y cualitativa) ha de responder a todos los aspectos formativos de los estudiantes.

\section{Conclusiones}

Con este trabajo hemos podido constatar la correcta compatibilidad de técnicas de análisis cualitativo y cuantitativo de datos en la realización de estudios como el que aquí se ha presentado. Al mismo tiempo, las cuestiones aquí planteadas han venido a ser un referente donde los estudiantes han podido satisfacer la necesidad de dar una opinión crítica sobre el sistema universitario del que forma parte.

Tras la aplicación de la técnica del análisis de contenido, hemos podido advertir cuál ha sido la tendencia en la respuesta de los estudiantes encuestados, advirtiendo su compromiso con el instrumento de recogida de información, así como los rasgos que, desde su perspectiva, conforman una buena formación. Se han hecho patentes los males que la afectan y se han propuesto alternativas para su mejora. Al final, hemos obtenido datos para describir los elementos que conforma una Universidad de calidad.

De este modo, una buena formación universitaria ha de estar caracterizada por un componente personal y profesional que permita al alumnado desarrollarse socialmente a través de la adquisición de habilidades, actitudes y valores.

Sin embargo, las deficiencias que afectan a su formación se centran en unos planes de estudios sobrecargados de asignaturas, muchas de ellas innecesarias y un exceso de contenidos teóricos en detrimento de los prácticos. Del mismo modo, acusan una falta de atención por parte del profesorado y una ausencia de relación con el mismo.

Para corregir estas deficiencias, las propuestas son muy escasas y poco pragmáticas. Aún así, consideran que ha de mejorarse el sistema de prácticas de modo que éstas sean más 
profesionales, han de introducirse nuevas metodologías didácticas centradas en la orientación laboral y una renovación de los planes de estudios que tiendan a la especialización.

Estas manifestaciones se han visto validadas tras un estudio de regresión múltiple donde, el aspecto que mejor representa la calidad de una Universidad es la satisfacción del alumnado, un indicador de producto, manifestación de la política educativa actual en materia de evaluación institucional. A pesar de ser este el elemento que mejor define esa calidad por parte del alumnado, los estudiantes consultados expresan la necesidad de disponer de un profesorado que les atienda, les motive y les proporcione, en una institución dotada de buenas instalaciones y recursos adecuados, una formación integral para su desenvolvimiento futuro en el ámbito social y en el mundo profesional, necesitada de mecanismos de orientación laboral y adecuados sistemas de evaluación del rendimiento académico.

Estos datos coinciden con los resultados aportados por el análisis discriminante, a pesar de que, repetida esta técnica clasificando a los estudiantes encuestados en función del rendimiento académico y la rama de especialización de los estudios en curso, ofrecen perfiles un tanto particulares de lo que, consideran, ha de concebirse como una Universidad de calidad. 


\section{Referencias}

Bartolomé, M. (1990). Elaboración y análisis de datos cualitativos. Aplicación a la investigación. Barcelona: Universidad de Barcelona.

Cantón, I. (Coord.) (2001). La implantación de la calidad en los centros educativos. Una perspectiva aplicada y reflexiva. Madrid: CCS.

CIGAL (2000). Premio Deming. http://www.cigal.igatel.net/html/p_deming.htm (15 de enero de 2003).

Consejo de Universidades (1998). Plan Nacional de Evaluación de la Calidad de las Universidades. Guía de evaluación. Madrid: Consejo de Universidades.

De la Orden, A. (1988). La calidad de la educación. Bordón, 40 (2), 149-161.

De Miguel, M. y Rodríguez Espinar, S. (1991). La evaluación de las instituciones universitarias. Madrid: Consejo de Universidades.

Doherty, G.D. (1994). Developing quality systems in education. Londres: Routledge. EFQM, (2002). Excellence Model. http://www.efqm.org/new_website/model_awards/model/excellence_model.htm (16 de enero de 2003).

Etxeberría, J. (1999). Regresión múltiple. Madrid: La Muralla / Salamanca: Hespérides.

Gazïel, H. et al. (2000). La calidad en los centros docentes del siglo XXI. Propuestas y experiencias prácticas. Madrid: La Muralla.

Grupo Helmántica (1995). Interacción profesor-alumno: una dimensión para la evaluación docente del profesorado Universitario. Actas del Congreso Nacional sobre Orientación y Evaluación Educativas. A Coruña: Universidade da Coruña.

Guillén, M.F. (1992). Análisis de regresión múltiple. Madrid: Centro de Investigaciones Sociológicas.

López, M. (1999). A la calidad por la evaluación. La evaluación de centros docentes. Madrid: Escuela Española.

Marchesi, A. y Martín, E. (1998). Calidad de la enseñanza en tiempos de cambio. Madrid: Alianza.

MECD (2001). Acciones de mejora para la calidad universitaria. Madrid: MECD.

OCDE (1991). Escuelas y calidad de la enseñanza. Informe internacional. Barcelona: Piados / Ministerio de Educación y Ciencia.

Pérez Juste, R. et al. (2000). Hacia una educación de calidad. Gestión, instrumentos y evaluación. Madrid: Narcea. 
Quintanilla, M.A. (1998). En pos de la calidad: notas sobre una nueva frontera para el sistema universitario español. Revista de Educación, 315, 85-95.

Rodríguez, G. et al. (1995). Análisis de datos cualitativos asistido por ordenador: AQUAD y NUDIST. Barcelona: PPU.

Sánchez, M.C., Prado, J.D. y Martín, M.A. (2001). Estudio cualitativo de los temas transversales implícitos en los libros de texto de lecturas utilizados en educación primaria. En AIDIPE. X Congreso Nacional de Modelos de Investigación Educativa. Investigación y evaluación en la sociedad del conocimiento (pp. 217-221). A Coruña: Universidade da Coruña.

Tejedor, F.J. (2003). Un modelo de evaluación del profesorado universitario. Revista de Investigación Educativa, 21 (1), 157-182. 